\title{
Italique
}

Poésie italienne de la Renaissance

III | 2000

Varia

\section{Tasso in Ottieri}

\section{Federico Sanguineti}

\section{OpenEdition}

\section{Journals}

Edizione digitale

URL: http://journals.openedition.org/italique/193

DOI: 10.4000/italique.193

ISSN: 1663-4438

\section{Editore}

Librairie Droz

\section{Edizione cartacea}

Data di pubblicazione: 31 dicembre 2000

Paginazione: 103-110

ISBN: 2-600-00490-4

ISSN: 1423-3983

\section{Notizia bibliografica digitale}

Federico Sanguineti, «Tasso in Ottieri », Italique [Online], III | 2000, online dal 06 octobre 2009 consultato il 23 avril 2019. URL : http://journals.openedition.org/italique/193 ; DOI : 10.4000/ italique.193 
FEDERICO SANGUINETI

T A S S O I N O T T I E R I 


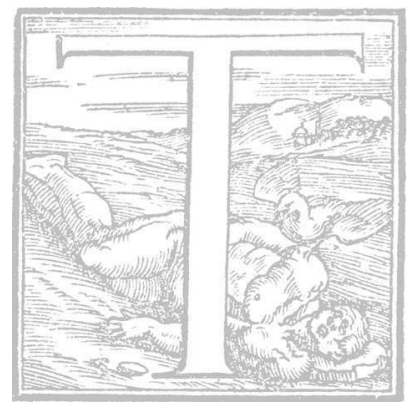

Asso «è stato l'Autore per molti e per molto tempo; ba goduto (almeno il passato prossimo è necessario, non altrettanto, temo, il presente) di fortuna ottima ed abbondante sul piano dell'auctoritas... Non sono più pochi ormai gli studi che negli ultimi decenni hanno permesso di decifrare, anche in pieghe assai riposte, le tracce della presenza di Torquato negli scrittori di mezzo mondo per quattro ininterrotti secoli, fino ai giorni nostri $»$. In un contributo dedicato a La fortuna editoriale tassiana dal 'soo ai giorni nostri, a queste considerazioni Lorenzo Carpanè non mancava di allegare qualche precisazione, osservando che « echi tassiani sono stati rilevati non solo nei poeti ottocenteschi, non solo in quelli di inizio secolo, ma anche in quelli contemporanei, come Parronchi o Caproni $\gg:{ }^{1}$ rinviando, in particolare, all'inchiesta condotta da Franco Di Carlo. ${ }^{2}$ Che riferimenti a personaggi del Tasso ("e tu in semiluce con Armida... »), unitamente a citazioni dall'episodio di Tancredi e Clorindapagina su cui si dovrà ritornare - siano reperibili nella poesia di Zanzotto, lo ricorda Tecla Gaio nel quinto "Quaderno di Filologia, Lingua e Letteratura Italiana» curato da Gilberto Lonardi. ${ }^{3}$ Né si può ignorare l'intervento dedicato, dal compianto Varanini, a Ungaretti lettore dell'ottava I3 del canto XVI della Gerusalemme Liberata, descrittiva di un pappagallo sudamericano, il "mirabil mostro» su cui, indagandone le fonti, anche Elisabetta Selmi si è soffermata. ${ }^{4}$

Vero è che la fortuna novecentesca rimane terreno di ricerca non fra $i$ più frequentati, e passibile magari di qualche sviluppo. Ne è prova l'individuazione, da parte di Antonio Zollino, per la Siepe di Pascoli, di un «illustre precedente tematico » e intertestuale in tre madrigali delle Rime amorose. $M a$, a dimostrazione di come il poeta della Gerusalemme sia "ritornato ad essere oggetto... di poesia ", Carpanè non poteva che additare, per scrupolo bibliografico, il poemetto Il sogno di Torquato Tasso, di Guerino D'Alessandro (199I), degno di nota "non tanto per il suo eventuale valore intrinseco, ma soltanto come segno comunque di attenzione, anche ai nostri tempi, per il poeta sorrentino ${ }^{6}{ }^{6}$

Malinconica conclusione, a cui non occorre rassegnarsi, giacché, documento di vitale presenza di Tasso nella letteratura contemporanea, soccorre l'ultimissima stagione di Ottiero Ottieri. ${ }^{7}$ Uscito nel 1996, il Poema osceno si presenta, fra l'altro, come spregiudicata rivisitazione della tradizione culturale italiana, condotta da un punto di vista che, con parola cara a Ferroni, si potrebbe definire "postumo». E "prova d'amore postuma» è additabile sia detto en passant - "nel lamento di Erminia rifugiatasi tra i pastori, che 
pensa alla propria tomba visitata dall'amato Tancredi $\gg .{ }^{8}$ Fatto sta che in uno dei dialoghi fra Vera e Pietro, al centro stesso del Poema di Ottieri, la donna non manca di richiamare alla mente del riluttante protagonista il modello testamentario di Tasso :

... oggi viene il notaio. Facciamo testamento insieme.

Pietro : Fallo tu, io non lo faccio.

Vera : Ma tu chi sei ? Anche il Tasso lo fece.

Nel cuore del Poema, Tasso si rivela pertanto modello cruciale, alter ego del protagonista. Due, si rammenti, sono i momenti intorno a cui ruota il prosimetro. Da un lato l'intermittente riferimento al panorama culturale odierno (o quasi) : ecco, ad esempio, i richiami a Maria Corti (p. I82), a Gadda "poetico e preciso 》 (p. I83), al « giornalista » Corrado Stajano (p. 204), al « filosofo » Gentile (p. 206), a Rebora « dimesso dal manicomio » (p. 224), a Pasolini « un po' irresponsabile 》 (p. 25I), a Zanzotto (p. 285), a Volponi (p. 386) e a Balestrini ( $p .423)$; né manca, implicitamente evocata, Susanna Tamaro di Va' dove ti porta il cuore (p. 375 e p. 495). Al polo opposto, da sempre ricorrente nel linguaggio e nell'opera di Ottieri, la presenza di Dante, ossessione che fa da sfondo al mito tassiano.

Troppo facile sarebbe insistere sulle esperienze esistenziali - Sant'Anna per Torquato, il «campo di concentrazione » per Ottiero - che avvicinano i due poeti. Difficile dire fino a che punto al figlio di Bernardo alludano questi versi: «Se un poeta vuole poetare / come un altro / superiore e famoso, / non può che torcersi / sul pavimento, insensato » (p. 432). Ineludibile appare però la confessione seguente, in cui l'esplicito paradigma dantesco e quello implicitamente tassiano si fondono indissolubilmente:

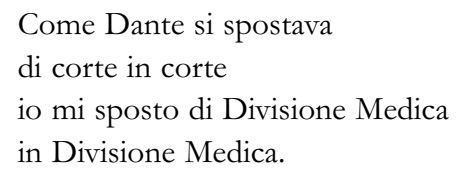

L'Alighieri dunque, con prelievi soprattutto dalla Vita Nova, infernali e paradisiaci. Si pensi, minimo specimen, al sonetto A ciascun'alma presa, trascritto adespoto nel Poema osceno $(p .478)$; 0 all'invocazione alla morte precedente Donna pietosa, "Dolcissima morte, vieni a me », cui si allude nei Due amori ("vieni dolce morte », p. 2I); o a Tanto gentile II, ancora una volta nei Due amori ("Che intender non lo può chi non lo prova", p. I0). Ed eccoci all'Inferno : il tòpos della "diritta via», presente in Tempi stretti (" uno che ba smarrito la retta via », p. 204), quindi in uno dei 
dialoghi di Di chi è la colpa ("Io mi trovo per una selva oscura non soltanto nel mezzo del cammin della mia vita, ma ogni due anni circa, o tutte le sere », p. I43), e finalmente nel Poema osceno ("Nell'ormai lungo cammino della vita », p. 427). Inoltre, prelievo da Inf. I, 90, "tremar le vene e i polsi $»$, in Tempi stretti ("Questo fa tremare le vene ai polsi della nostra Direzione ", p. II4) ; oppure, dal v. II3, " seconda morte », nel Poema osceno (" morte seconda 》, p. 92). Più rapidamente : canto quarto, 84, "sembianza avean né trista né lieta», cui si allude, con minima variatio, nelle Memorie dell'incoscienza ("Non avevano un aspetto triste, né furioso 》, p. I73); verso 104, "'l tacere è bello", recuperato nel Poema osceno ("il tacer mi è bello », p. I44). Canto quinto, emistichio dal v. 25, "le dolenti note », ripreso nel Poema osceno (p. 297); v. 86, "aere maligno », già in Tempi stretti ("soffiava un'aria maligna", p. 207). E via discorrendo. Si potrebbe proseguire ricordando che allusivo al « disdegnoso gusto » di Inf. I3, 70 è fin il titolo di una sezione della Corda corta, Il gusto del disgusto ; o che il quesito di Inf. Is, 46, "qual fortuna o destino? ", è amplificato ne Il palazzo e il pazzo ("... qual causa, qual fine, qual fuga, / espulsione, sofferenza, gioco mortale, alternativa », p. 8I); o che l'" arco sesto 》 di Inf. 2I, 108 anticipa, forse, l'« orgasmo sesto » nel Poema osceno ( $p .57)$; fino a riconoscere, nella pagina di Ottieri, magari pasolinianamente mediato, il lessico dell'ultima cantica : "trasumanar» di Par. I, 70 ("stufa di organizzare, voglio / trasumanare 》, p. 229; 《 organizzar trasumanar 》, p. 397); «infuturo » di Par. 17,98 ("Ma, oggi, / non m'infuturo», p. 175); non senza il recupero di identica clausola (" tanto disio» di Par. 28, I30, insieme all'ovvio ricordo di Inf. 5, II3, "quanto disio ») in versi dove sembra oltretutto risuonare un'eco della Gerusalemme ("... sempre alla fretta / stimol novo s'aggiunge e cagion nova ", XII 563-4): "Non capi che l'amplesso aveva / tanta fretta, in sé, tanto desio, / che l'amplesso avvenia prima d'amplesso » (P. 3I). Dalla seconda cantica nulla o quasi, a parte il reiterato richiamo al « fren de l'arte » del canto conclusivo del Purgatorio, v. I4I, da un lato chiosato come "fren della vita» ("Non trovo... / il minimo accenno / ... a una minima funzione / anti-età, anti-morte / della gloria eventuale, / del fren dell'arte, / del fren della vita ", $p .378) ; e$, d'altro canto, posto a confronto con un ipotetico "fren d'amore»( protagonista :

Ci dobbiamo abbandonare alla piena dell'amore. Non esiste un fren d'amore come, nell'arte, il fren dell'arte.

Romantiche parole che presuppongono uno scontro, si direbbe, senza freni e non privo di implicazioni erotiche, dove "l'uso de l'arte» viene appunto 
impedito da « ombra 》 e " furore 》: ancora una volta il dodicesimo canto della Gerusalemme. Del resto, l'invocazione a una notte, come ha scritto Arnaldo Di Benedetto, "emblematica del cieco "furore »... che impronta le azioni ${ }^{9}$ di Tancredi e Clorinda (Liberata XII 57), sembra rivivere nell'incipit del poemetto intitolato Gli altri o le proiezioni invidiose :

\author{
O notte da Milano a Parigi, \\ ultima spiaggia! Notte nel vagone \\ letto dove non ti spogliasti e il \\ dottorino ti dava un barbiturico l'ora. \\ O discesa alla Gare de Lyon...
}

Ma si pensi ancora alla prima sezione della Corda corta, Alle betulle, dove la memoria del ritiro "stanco e anelante» dei combattenti (XII 57) traspare nell'endecasillabo

come un guerriero vincitore e stanco.

Insomma, chi se non Ottieri poteva far si che Tancredi ritornasse sulla scena, con abiti borghesi, in un presente neobarocco, dove la « sanguigna e spaziösa porta» del Tasso (XII 62 5) diviene impraticabile (né può sfuggire, in «ami il bersaglio amato », l'eco di «Abi! tanto amò la non amante amata», II $288)$ :

\author{
O lei, o morte, \\ senza sanguinosa pugna, \\ armistizio dalla durata incerta. \\ $\cdots$ \\ Tancredi, sei una sagoma. \\ Mercedes, Mercedes, \\ BMW, Fuoristrade. \\ Tancredi, l'anima \\ non ami, ami la sagoma; \\ non i belli dentro. \\ Ami il bersaglio amato \\ della carabina computerizzata e olimpica, \\ senza polvere da sparo. \\ Con te vanno i tempi, \\ ma tu con i tempi vai.
}


In siffatta riduzione del cavaliere cristiano a "sagoma » sembra denunciata quella condizione che, a proposito di Flaubert, Sartre ba chiamato " costituzione passiva ». Dove mai, c'è infine da domandarsi, se non nei versi del Seduttore passivo, pubblicato nel 1995 (guarda caso, il centenario della morte di Tasso), sarà dato reperire adeguato omaggio, da parte di un poeta vivente, all'autore della Gerusalemme? La celebrazione non può assumere altra forma, per Ottiero Ottieri, che quella di un'allucinata rievocazione, paradosso nel paradosso, del già ricordato combattimento di Tancredi e Clorinda :

Tancredi intanto

va di Clorinda

ad aspettar l'assalto,

passivo.

Non è facile scrivere

sotto giudizio frustrante,

sull'impressione

che la terza età

non ha pietà.

Per un mese, per un anno,

siede alle cinque,

Tancredi al bar,

con caffé freddo.

Federico Sanguineti 
FEDERICO SANGUineti

I. L. Carpanè, La fortuna editoriale tassiana dal 'soo ai giorni nostri, «Italianistica », 24 (I995), 2-3, p. $54 \mathrm{I}$.

2. F. Di Carlo, Ungaretti e Leopardi. Il sistema della "memoria» dall' "assenz̧a » all' innocenza », Bulzoni, Roma, I979; Id., Invito alla lettura di Tasso, Milano, Mursia, I990, pp. I42-50.

3. T. Gaio, Nome di dea, invocata virtù. Un percorso nella poesia di Andrea Zanzotto, "Quinto Quaderno di Filologia, Lingua e Letteratura Italiana », Università di Verona, I996, p. I67 e n. 4 .

4. G. Varanini, Ungaretti fra il «papagai» di Don Denis e il «mirabil mostro» del Tasso, in Humanitas e Poesia. Studi in onore di Gioacchino Paparelli, a c. di L. Reina, Salerno, Laveglia, I 990, pp. 945-55; E. Selmi, Il « mirabil mostro » del giardino di Armida fra « esemplarità » retorica ed esotismo americano, "Studi Tassiani », 4I (I993), pp. I 5 7-7 I.

5. A. Zollino, Tasso e D'Annunz̧io nella « Siepe » di Pascoli, «Lettere Italiane », 48 (I996), I, pp. 95-100.

6. L. Carpanè, Rassegna bibliografica degli studi tassiani (I99I), "Studi Tassiani », 42 (I994), p. I6I.

7. Le opere di Ottieri sono citate dalle seguenti edizioni : Memorie dell'incoscienza, Milano, Bompiani, I967 [I ${ }^{\mathrm{a}}$ ediz. Torino, Einaudi, I954] ; Tempi stretti, Torino, Einaudi, I964; La corda corta, Milano, Bompiani, I 978 ; Di chi è la colpa, Milano, Bompiani, I 979; I due amori, Torino, Einaudi, I993; Il palazzo e il pazzo, Milano, Garzanti, I993; Il seduttore passivo, Firenze, Giunti, I 995 ; Il poema osceno, Milano, Longanesi, I 996.

8. G. Ferroni, Dopo la fine. Sulla condizione postuma della letteratura, Torino, Einaudi, I 996, pp. 7I-2 e n. 35 .

9. A. Di Benedetto, Un esempio di poesia tassiana (il canto XII della "Gerusalemme Liberata»), «Studi Tassiani », 4I (I993), p. 238. 